\title{
Speckle photography with different pupils in a multiple-exposure scheme
}

\author{
Luciano Angel, ${ }^{*}$ Myrian Tebaldi, ${ }^{\dagger}$ Néstor Bolognini, ${ }^{\ddagger}$ and Marcelo Trivi ${ }^{\dagger}$ \\ Centro de Investigaciones Ópticas (Consejo Nacional de Investigaciones Científicas y Técnicas-Comisión \\ de Investigaciones Científicas) and Optimo, Departamento de Fisicomatemática, Facultad de Ingeniería, \\ Universidad Nacional de la Plata, La Plata, Argentina, C.C. 124, Correo Central, 1900 La Plata, Argentina
}

Received April 29, 1999; revised manuscript received August 31, 1999; accepted September 27, 1999

\begin{abstract}
The use of different multiple-aperture pupils for recording each image in speckle photography is proposed. The introduction of suitable spatial frequency carriers, by internally modulating imaged speckles, allows one to selectively isolate or combine the spectral content of different images into spatially separated regions in the Fourier plane. Theoretical and experimental results extend the speckle photography technique to the depiction of several specklegrams of multiple uniform in-plane displacements. In this case, because different pupils are considered for recording, the cross-correlation functions for the amplitudes and intensities in the image plane are calculated on the basis of the statistical properties of the object. Also, the ensemble-average intensity in the Fourier plane is analytically derived, and fringe visibility is investigated. () 2000 Optical Society of America [S0740-3232(00)01701-4]

OCIS codes: $120.0120,120.3180,120.3940,120.6160$.
\end{abstract}

\section{INTRODUCTION}

Speckle photography is a well-known informationprocessing technique in which speckle displacement by coherent optical processing is determined. ${ }^{1}$ It is based on the introduction of spatial frequency carriers through speckle modulation, which allows the low-frequency spectral components of images to be spread out into the highfrequency region of the Fourier-transform plane. As in other multiplexing approaches to optical image processing and speckle metrology, ${ }^{1}$ speckle photography combines speckle modulation and displacement. Speckle photography uses an essentially simpler optical arrangement, has a wider range of measurement, and has lesssevere stability requirements than for holographic and speckle interferometry.

Speckle photographic techniques have been implemented in the diffraction field (free-space geometry) and in image configurations, to which only a limited area of the object under illumination contributes. ${ }^{2}$ An image obtained through double or multiple displacement of a diffuse object that is generating speckles is recorded. Then the specklegram is analyzed through coherent illumination and Fourier transformation of transmitted light. Two methods ${ }^{3}$ are available for speckle photography. One of them consists of a addressing a narrow laser beam on a specklegram point-by point to form Young fringes, whose orientation and spacing can be interpreted in terms of the direction and the magnitude of the diffuser displacement. The orientation of these fringes is normal to the direction of speckle displacement, whereas their spacing is inversely proportional to the magnitude of the displacement. The other method is based on the spatial filtering of a specklegram, which yields the contour lines of speckle displacement along a direction that depends on the position of the filtering aperture in the Fourier plane.

When multiple exposures are made, each of the fields has a correlation area that corresponds to the mean size of the speckles, and object deformation between exposures gives rise to both displacement and decorrelation of laser speckles. Speckle displacement and changes in structure have been investigated by calculation of the crosscorrelation functions of speckle intensities before and after displacement. Specifically, the dependence of the speckle correlation properties on the conditions of object illumination and speckle observation as well as on the displacement parameters was theoretically analyzed. ${ }^{2-6}$

To measure accurately the periodicity of the Young fringes obtained in speckle photography, the intensity profile of the diffraction halo is analyzed. ${ }^{6,7}$ In an image configuration, the form of the diffraction halo is determined by the aperture function of the recording lens. If the intensity distribution of the imaged speckle pattern is uniform, ergodic, and Gaussian, then the diffraction halo is equal to the power spectrum of the image-plane speckle intensity. $^{7}$

Although an optical system with a single-aperture pupil is usually employed to form the images, some advantage is achieved in speckle photography by use of a multiple-aperture pupil.8,9 Assuming that the total amount of diffracted light is the same from speckle patterns recorded by a single aperture and by multiple apertures, the amount of diffracted light at the high frequencies selected by the multiple apertures is much greater than the corresponding values from single-aperture recording. Thus multiple apertures yield an increased signal-to-noise ratio and consequently much better fringe resolution.

In this paper we propose the use of different multipleaperture pupils for recording each image in speckle photography. To our knowledge, this technique was not previously investigated. As we show, this approach has a definite advantage in that it allows selective concentra- 
tion of the spectral components of individual or various speckled images into isolated spots in the Fourier plane. On this basis, a remarkable extension of speckle photography to depict in a single frame several systems of interferometric fringes for different uniform in-plane displacements can be implemented.

When different pupils are employed to form the images, the decorrelation introduced by changing the pupils between exposures must be analyzed. Then the crosscorrelation functions of speckle amplitudes and intensities in the image plane are evaluated in terms of the geometric characteristics of the pupils. Finally, expressions for the observed average intensity and fringe visibility in the Fourier plane are derived. Moreover, exact knowledge of these correlation properties is used to optimize fringe visibility.

The fundamentals and the relative benefits of the proposed approach are discussed on the basis of the theoretically predicted features of fringe-modulated diffraction patterns. Also, experimental results that support our analysis are presented.

\section{THEORETICAL ANALYSIS}

\section{A. Diffuser Properties and Image Formation}

Let us consider the experimental setup of Fig. 1(a). A random diffuser, located at the input plane $x-y$, is illuminated by a collimated laser beam of wavelength $\lambda_{W}$ and intensity $I_{W}$. This beam impinges perpendicularly on the mean (or smoothed) diffuser surface.

The amplitude transmission function of the diffuser is given by $\zeta(x, y) \exp [i \phi(x, y)]$, where $\zeta(x, y)$ is the macroscopic transmission function and $\exp [i \phi(x, y)]$-is the microscopic one, which is a random phasor related to the diffusing properties of the object. For most diffuser objects, except for observation in the region where the directly transmitted or specularly reflected component is superimposed on the diffused component, the following conditions

(a)

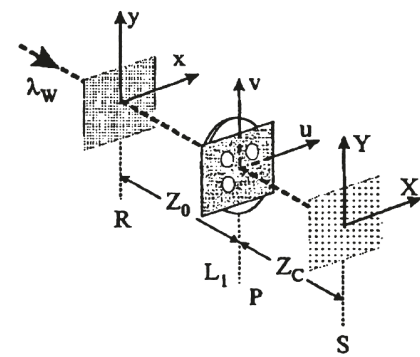

(b)

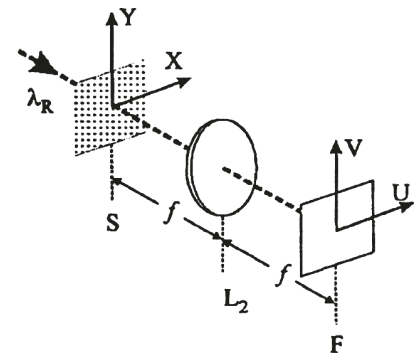

Fig. 1. Experimental setups for (a) recording and (b) analysis of the specklegram. $R$, diffuser; $L_{1}$, imaging lens; $P$, pupil mask; $S$ 's, specklegrams; $L_{2}$, Fourier lens; F, Fourier plane. are satisfied ${ }^{3}$ : The phases $\phi(x, y)$ are uniformly distributed over the interval $(-\pi, \pi)$ (i.e., the surface is rough compared with the wavelength) and the phases for two different points are statistically independent of each other (i.e., the elementary scattering areas are unrelated). In addition, the intensity transmission function $|\zeta(x, y)|^{2}$ has a constant value. In practice, 0 $<|\zeta(x, y)|^{2}<1$.

The field amplitude immediately in front of the diffuser, at a time $t^{k}$, is given by

$$
\begin{aligned}
A_{0}^{k}(x, y)= & \sqrt{I_{W}} \zeta\left(x-x^{k}, y-y^{k}\right) \\
& \times \exp \left[i \phi\left(x-x^{k}, y-y^{k}\right)\right],
\end{aligned}
$$

where the vector $(x, y)$ denotes a generic point on the diffuser plane and the vector constant $\left(x^{k}, y^{k}\right)$ determines the position of the diffuser at that time. This vector in general varies from one exposition to another according to the diffuser displacement.

An image of this input is formed in the $X-Y$ plane by use of a lens $\mathrm{L}_{1}$ (Fig. 1) located at the $u-v$ plane. To image the input $A_{0}^{k}(x, y)$, a dark stop $P^{k}$ with several apertures is located immediately in front of the lens. This pupil is represented by the function $P^{k}(u, v)$, which is unity inside the apertures and zero otherwise. Then the image field amplitude is proportional to

$$
A^{k}(X, Y)=\int A_{0}^{k}(x, y) K^{k}(X, Y ; x, y) \mathrm{d} x \mathrm{~d} y,
$$

where

$$
\begin{aligned}
K^{k}(X, Y ; x, y)= & \int P^{k}(u, v) \exp \left\{-i \frac{2 \pi}{\lambda_{W}}\left[\left(\frac{x}{Z_{0}}+\frac{X}{Z_{C}}\right) u\right.\right. \\
& \left.\left.+\left(\frac{y}{Z_{0}}+\frac{Y}{Z_{C}}\right) v\right]\right\} \mathrm{d} u \mathrm{~d} v
\end{aligned}
$$

is the impulse response of the optical system for the mean object surface. The distances from the diffuser to the lens and from the lens to the image plane are $Z_{0}$ and $Z_{C}$, respectively.

\section{B. Cross-Correlation Functions for the Image Amplitudes and Intensities}

Because of the inherent statistical nature of the experimental situation presented in Subsection 2.A, introduction of the statistical optics formalism is best suited for treating the present problem. Accordingly, the crosscorrelation functions for the image amplitudes and intensities are calculated, in terms of which the change in the diffusely transmitted light caused by object displacement can be described.

To this end, let us consider that $A^{k}(X, Y)$ and $A^{l}(X, Y)$ represent the image amplitude fields for, in general, two different positions of the diffuser, which are denoted $\left(x^{k}, y^{k}\right)$ and $\left(x^{l}, y^{k}\right)$. Also, these images are obtained in general by using two different pupils, $P^{k}(u, v)$ and $P^{\prime}(u, v)$, respectively.

Then, from Eqs. (1) and (2), we obtain the crosscorrelation function of the image amplitudes: 
$\left\langle A^{k}\left(X_{a}, Y_{a}\right)\left[A^{l}\left(X_{b}, Y_{b}\right)\right]^{*}\right\rangle$

$$
\begin{aligned}
\approx & I_{W}|\zeta|^{2} \int K^{k}\left(X_{a}, Y_{a} ; x+x^{k}, y+y^{k}\right) \\
& \times\left[K^{l}\left(X_{b}, Y_{b} ; x+x^{l}, y+y^{l}\right)\right]^{*} \mathrm{~d} x \mathrm{~d} y,
\end{aligned}
$$

where the statistical properties of the elementary phasors and the uniformity of the intensity transmission function of the diffuser were considered. The average procedure <) that is performed over a statistical ensemble of the microscopic structure of the object is introduced to eliminate the speckle-like noise, and it can be physically interpreted as a spatial average over a region of a sufficient extent. ${ }^{3}$

Furthermore, substituting Eq. (3) into Eq. (4) yields where

$$
\begin{gathered}
P^{k l}(u, v) \equiv P^{k}(u, v) P^{l}(u, v), \\
\left(\Delta x^{k l}, \Delta y^{k l}\right) \equiv\left(x^{l}-x^{k}, y^{l}-y^{k}\right),
\end{gathered}
$$

and $\mathfrak{F}$ indicates a two-dimensional Fourier transform.

Because $\left(x^{k}, y^{h}\right)$ and $\left(x^{l}, y^{h}\right)$ represent different positions of the diffuser when it is forming the respective images, it follows that the vector $\left(\Delta x^{k l}, \Delta y^{k I}\right)$ stands for the relative uniform in-plane displacement that the diffuser undergoes between images. Note that we consider this vector when evaluating the Fourier transform in relation (5b), which implies that the correlation of the field ampli-

$\left\langle A^{k}\left(X_{a}, Y_{a}\right)\left[A^{l}\left(X_{b}, Y_{b}\right)\right]^{*}\right\rangle$

$$
\begin{aligned}
\approx & \int\left(\int P^{k}(u, v) \exp \left\{-i \frac{2 \pi}{\lambda_{W}}\left[\left(\frac{x^{k}}{Z_{0}}+\frac{X_{\mathrm{a}}}{Z_{C}}\right) u+\left(\frac{y^{k}}{Z_{0}}+\frac{Y_{a}}{Z_{C}}\right) v\right]\right\} \exp \left[-i \frac{2 \pi}{\lambda_{W} Z_{0}}(x u+y v)\right] \mathrm{d} u \mathrm{~d} v\right. \\
& \left.\times \int P^{\prime}\left(u^{\prime}, v^{\prime}\right) \exp \left\{i \frac{2 \pi}{\lambda_{W}}\left[\left(\frac{x^{\prime}}{Z_{0}}+\frac{X_{b}}{Z_{C}}\right) u^{\prime}+\left(\frac{y^{\prime}}{Z_{0}}+\frac{Y_{b}}{Z_{C}}\right) v^{\prime}\right]\right\} \exp \left[i \frac{2 \pi}{\lambda_{W} Z_{0}}\left(x u^{\prime}+y v^{\prime}\right)\right] \mathrm{d} u^{\prime} \mathrm{d} v^{\prime}\right) \mathrm{d} x \mathrm{~d} y \\
\approx & \iint\left(\int \exp \left\{-i \frac{2 \pi}{\lambda_{W} Z_{0}}\left[x\left(u-u^{\prime}\right)+y\left(v-v^{\prime}\right)\right]\right\} \mathrm{d} x \mathrm{~d} y\right. \\
& \times P^{k}(u, v) \exp \left\{-i \frac{2 \pi}{\lambda_{W}}\left[\left(\frac{x^{k}}{Z_{0}}+\frac{X_{a}}{Z_{C}}\right) u+\left(\frac{y^{k}}{Z_{0}}+\frac{Y_{a}}{Z_{C}}\right) v\right]\right\} \\
& \left.\times P^{\prime}\left(u^{\prime}, v^{\prime}\right) \exp \left\{i \frac{2 \pi}{\lambda_{W}}\left[\left(\frac{x^{l}}{Z_{0}}+\frac{X_{b}}{Z_{C}}\right) u^{\prime}+\left(\frac{y^{I}}{Z_{0}}+\frac{Y_{b}}{Z_{C}}\right) v^{\prime}\right]\right\}\right) \mathrm{d} u \mathrm{~d} v \mathrm{~d} u^{\prime} \mathrm{d} v^{\prime} .
\end{aligned}
$$

Note that the walk-off of the object points out of the illuminated region causes speckle decorrelation. This effect is included when we consider a finite integration region over $x$ and $y$ in relation (5a). The scope of our analysis concerns mainly those effects that refer to the configuration of the multiple-aperture pupils. To study the decorrelation that is due only to pupil changes between exposures, in the following we disregard the finite dimensions of the diffuser illuminated area. If we assume that the illumination area is large enough, then

$$
\begin{aligned}
\int \exp \left\{-i\left(2 \pi / \lambda_{W} Z_{0}\right)\left[x\left(u-u^{\prime}\right)+\right.\right. & \left.\left.y\left(v-v^{\prime}\right)\right]\right\} \mathrm{d} x \mathrm{~d} y \\
& =\delta\left(u-u^{\prime}, v-v^{\prime}\right) .
\end{aligned}
$$

and relation $(5 \mathrm{a})$ reduces to tudes is affected by the diffuser displacement.

The function $P^{k l}(u, v)$ can be associated with a pupil $P^{k J}$ whose transmission area corresponds to the transmission area that $P^{k}$ has in common with $P^{\prime}$. In the following, the function $P^{k l}$ is referred to as the common part of $P^{k}$ and $P^{\prime}$.

According to relation (5b), the correlation between two speckled images obtained through two different pupils is determined by the common part of the respective pupils and displacements. In particular, if there are no common transmission areas in the pupils $\left[P^{k l}(u, v)=0\right]$, then the speckle patterns are completely uncorrelated; that is, $\left\langle A^{k}\left(X_{a}, Y_{a}\right)\left[A^{l}\left(X_{b}, Y_{b}\right)\right]^{*}\right\rangle=0$. As expected, this equation demonstrates that the complex amplitudes of waves going through different apertures are statistically independent of one another, because different components of the angular spectrum of scattered light are accepted by the different apertures.

$$
\begin{aligned}
\left\langle A^{k}\left(X_{a}, Y_{a}\right)\left[A^{l}\left(X_{b}, Y_{b}\right)\right]^{*}\right\rangle \approx & \iint P^{k}(u, v) P^{l}\left(u^{\prime}, v^{\prime}\right) \exp \left\{-i \frac{2 \pi}{\lambda_{W}}\left[\left(\frac{x^{k}}{Z_{0}}+\frac{X_{a}}{Z_{C}}\right) u-\left(\frac{x^{l}}{Z_{0}}+\frac{X_{b}}{Z_{C}}\right) u^{\prime}\right]\right\} \\
& \times \exp \left\{-i \frac{2 \pi}{\lambda_{W}}\left[\left(\frac{y^{k}}{Z_{0}}+\frac{Y_{a}}{Z_{C}}\right) v-\left(\frac{y^{l}}{Z_{0}}+\frac{Y_{b}}{Z_{C}}\right) v^{\prime}\right]\right\} \delta\left(u-u^{\prime}, v-v^{\prime}\right) \mathrm{d} u^{\prime} \mathrm{d} v^{\prime} \mathrm{d} u \mathrm{~d} v \\
\approx & \mathfrak{F}\left\{P^{k l}(u, v)\right\}\left(\frac{X_{a}-X_{b}}{\lambda_{W} Z_{C}}-\frac{\Delta x^{k l}}{\lambda_{W} Z_{0}}, \frac{Y_{a}-Y_{b}}{\lambda_{W} Z_{C}}-\frac{\Delta y^{k l}}{\lambda_{W^{\prime}} Z_{0}}\right),
\end{aligned}
$$


Now we evaluate the average intensity in the image plane by substituting the following for the same point $\left(X_{a}, Y_{a}\right)=\left(X_{b}, Y_{b}\right)$ in relation $(5 b)$ :

$$
\begin{aligned}
\left\langle I^{k}\right\rangle=\left\langle I^{k}(X, Y)\right\rangle & =\left\langle A^{k}(X, Y)\left[A^{k}(X, Y)\right]^{*}\right\rangle \\
& \approx \mathfrak{F}\left\{P^{k}(u, v)\right\}(0,0),
\end{aligned}
$$

where $\left(\Delta x^{k k}, \Delta y^{k h}\right)=0$ and $P^{k k}(u, v)=\left(P^{k}(u, v)\right.$ were used. Of course, $\left\langle I^{k}\right\rangle$ is a constant.

Moreover, the intensity cross-correlation function that describes the statistical properties of speckle patterns can be derived from the correlation functions of the complex amplitudes. If we assume that $A^{k}(X, Y)$ and $A^{l}(X, Y)$ obey the circular Gaussian statistics with zero mean in the complex plane, it follows that

$$
\begin{aligned}
& \left\langle I^{k}\left(X_{a}, Y_{a}\right) I^{I}\left(X_{b}, Y_{b}\right)\right\rangle \\
& =\left\langle I^{k}\right\rangle\left\langle I^{\prime}\right\rangle+\left|\left\langle A^{k}\left(X_{a}, Y_{a}\right)\left[A^{I}\left(X_{b}, Y_{b}\right)\right]^{*}\right\rangle\right|^{2},
\end{aligned}
$$

which is realized because the diffuser is rough compared with the wavelength ${ }^{10}$ and also because the correlation patch of the complex amplitude directly after being scattered by the object is much smaller than the resolution patch of observation. ${ }^{2}=4$

When the explicit expressions given by relation (5b) and Eq. (6) are substituted into Eq. (7), the crosscorrelation function for the intensities in the image plane is

$$
\begin{aligned}
&\left\langle I^{k}\left(X_{a}, Y_{a}\right) I^{l}\left(X_{b}, Y_{b}\right)\right\rangle \\
& \approx \mathfrak{F}\left\{P^{k}(u, v)\right\}(0,0) \mathfrak{F}\left\{P^{l}(u, v)\right\}(0,0) \\
&+\mid \mathfrak{F}\left\{P^{k l}(u, v)\right\}\left(\frac{X_{a}-X_{b}}{\lambda_{W} Z_{C}}-\frac{\Delta x^{k l}}{\lambda_{W} Z_{0}}, \frac{Y_{a}-Y_{b}}{\lambda_{W} Z_{C}}\right. \\
&\left.-\frac{\Delta y^{k l}}{\lambda_{W} Z_{0}}\right)\left.\right|^{2}
\end{aligned}
$$

Relation (8a) also can be written as
In the recording process, which is carried out by using the setup depicted in Fig. 1 (a), the intensity distributions $I^{k}(X, Y)=\left|A^{k}(X, Y)\right|^{2}$ for $k=1,2, \ldots N$ are sequentially recorded. The amplitude $A^{k}(X, Y)$ is given by Eqs. (1)(3), and a linear recording procedure is assumed for analytical purposes. ${ }^{4.6=9.11,12}$ Then the amplitude transmission function of the recorded specklegram can be assumed to be proportional to

$$
I(X, Y)=\sum_{k=1}^{N} I^{k}(X, Y) .
$$

In accordance with Fig. 1(b), in the analysis the specklegram is located in the $X-Y$ plane and illuminated by a collimated laser beam of wavelength $\lambda_{R}$ and intensity $I_{R}$, which impinges perpendicularly on the $X-Y$ plane. We assume a general case in which the wavelengths and intensities in the recording and the analysis steps are different; that is, $\lambda_{R} \neq \lambda_{W}$ and $I_{R} \neq I_{W}$. Afterward, the transmitted light is Fourier transformed by means of lens $\mathrm{L}_{2}$ (Fig. 1) of focal length $f$, and the intensity distribution is observed at the $U-V$ focal plane.

Thus the field amplitude in the transform plane is proportional to

$$
G_{f}(U, V)=\sqrt{I_{R}} \sum_{k=1}^{N} G^{k}(U, V),
$$

where

$$
G^{k}(U, V)=\int I^{k}(X, Y) \exp \left[-i \frac{2 \pi}{\lambda_{R} f}(X U+Y V)\right] \mathrm{d} X \mathrm{~d} Y
$$

On this basis, it follows that the cross correlation of the amplitudes in the Fourier plane is

$\left\langle I^{k}\left(X_{a}, Y_{a}\right) I^{I}\left(X_{b}, Y_{b}\right)\right\rangle \approx\left\langle I^{k}\right\rangle\left\langle I^{\prime}\right\rangle\left\{1+\frac{\left|\mathfrak{F}\left\{\left|P^{k l}(u, v)\right|^{2}\right\}\left(\frac{X_{a}-X_{b}}{\lambda_{W} Z_{C}}-\frac{\Delta x^{k l}}{\lambda_{W} Z_{0}}, \frac{Y_{a}-Y_{b}}{\lambda_{W} Z_{C}}-\frac{\Delta y^{k l}}{\lambda_{W} Z_{0}}\right)\right|^{2}}{\int\left|P^{k}(\xi, \chi)\right|^{2} \mathrm{~d} \xi \mathrm{d} \chi \int\left|P^{\prime}\left(\xi^{\prime}, \chi^{\prime}\right)\right|^{2} \mathrm{~d} \xi^{\prime} \mathrm{d} \chi^{\prime}}\right\}$,

which reduces to the well-known form of the autocorrelation function of the speckled intensity ${ }^{10}$ for $k=1$.

\section{Average Intensity Distribution of Diffracted Light} Another important function for describing the properties of speckle is the Fourier transform of the cross-correlation function, whose real part is related to the Young fringes observed in speckle photography.

In the following, the case of a multiple-exposure specklegram through different multiple-aperture pupils is considered. An expression for the average intensity in the Fourier plane is analytically derived on the basis of the statistical properties of recorded speckle distributions. $\left\langle G_{f}\left(U_{1}, V_{1}\right)\left[G_{f}\left(U_{2}, V_{2}\right)\right]^{*}\right\rangle$

$$
\begin{aligned}
= & I_{R} \sum_{k, I=1}^{N} \int\left\langle I^{k}\left(X_{a}, Y_{a}\right) I^{I}\left(X_{b}, Y_{b}\right)\right\rangle \\
& \times \exp \left(-i \frac{2 \pi}{\lambda_{R} f}\left(X_{a} U_{1}-X_{b} U_{2}+Y_{a} V_{1}\right.\right. \\
& \left.\left.-Y_{b} V_{2}\right)\right) \mathrm{d} X_{a} \mathrm{~d} Y_{a} \mathrm{~d} X_{b} \mathrm{~d} Y_{b} .
\end{aligned}
$$

Then, when we evaluate this cross correlation for $\left(U_{1}, V_{1}\right)=\left(U_{2}, V_{2}\right)$, it follows that the diffuser's ensemble-average intensity in the transform plane is 


$$
\begin{aligned}
\left\langle I_{f}(U, V)\right\rangle= & \left\langle G_{f}(U, V)\left[G_{f}(U, V)\right]^{*}\right\rangle \\
= & I_{R} \sum_{k, I=1}^{N} \int\left\langle I^{k}\left(X_{a}, Y_{a}\right) I^{l}\left(X_{b}, Y_{b}\right)\right\rangle \\
& \times \exp \left\{-i \frac{2 \pi}{\lambda_{R} f}\left[U\left(X_{a}-X_{b}\right)\right.\right. \\
& \left.\left.+V\left(Y_{a}-Y_{b}\right)\right]\right\} \mathrm{d} X_{a} \mathrm{~d} Y_{a} \mathrm{~d} X_{b} \mathrm{~d} Y_{b} .
\end{aligned}
$$

Also, the cross-correlation function of intensities can be expressed according to relation (5b) and $\mathrm{Eq}$. (7). Then, by substituting these expressions into Eq. (13), we obtain

$$
\begin{aligned}
\left\langle I_{f}(U, V)\right\rangle= & \delta(U, V)+\frac{1}{4} \int \mathrm{d}\left(X_{a}+X_{b}\right) \mathrm{d}\left(Y_{a}+Y_{b}\right) \\
& \times \sum_{k, I=1}^{N} \int \mid \mathfrak{F}\left\{P^{k l}(u, v)\right\} \\
& \times\left.\left(\frac{X^{\prime}+\Delta X^{k l}}{\lambda_{W} Z_{C}}, \frac{Y^{\prime}+\Delta Y^{k J}}{\lambda_{W} Z_{C}}\right)\right|^{2} \\
& \times \exp \left[-i \frac{2 \pi}{\lambda_{R} f}\left(U X^{\prime}+V Y^{\prime}\right)\right] \mathrm{d} X^{\prime} \mathrm{d} Y^{\prime},
\end{aligned}
$$

where $X^{\prime}=X_{a}-X_{b}, Y^{\prime}=Y_{a}-Y_{b}$, and for simplicity the constant factor $I_{R}$ was neglected. In Eq. (14), the vector

$$
\left(\Delta X^{k l}, \Delta Y^{k l}\right)=-\frac{Z_{C}}{Z_{0}}\left(\Delta x^{k l}, \Delta y^{k l}\right)
$$

is the relative displacement of $A^{l}(X, Y)$ with respect to $A^{k}(X, Y)$, where it was assumed that the speckle displacement takes place near the optical axis. The factor $-Z_{C} / Z_{0}$ represents the imaging magnification. The $\delta(U, V)$ term in Eq. (14) stands for a brilliant central point in the diffraction field produced by the constant value [see relations (6)] of the product $\left\langle I^{k}\right\rangle\left\langle I^{I}\right\rangle$. The constant factor $\frac{1}{4} \int \mathrm{d}\left(X_{a}+X_{b}\right) \mathrm{d}\left(Y_{a}+Y_{b}\right)$ represents the finite area of the specklegram. In the following, the $\delta(U, V)$ term and the area factor are not considered.

The diffuser displacement causes both speckle displacement and structural changes in the image plane. A double-exposed specklegram for a uniform in-plane displacement consists of many speckle pairs that are mutually independent with respect to shape and position. The two grains that belong to a speckle pair originally are similar but then are displaced from each other. Because of the finite dimensions of the specklegram, unpaired grains appear near the edge of the recording medium. The number of single grains increases as speckle decorrelation in proportion to the image displacement. ${ }^{13}$ Similar assertions can be made in connection with a multipleexposure scheme. In the following, it is assumed that the actual specklegram area is much larger than the characteristic speckle and displacement dimensions, which implies that the decorrelation effect associated with the diffuser displacement becomes negligible. In the process, the limits of the integrals over $X^{\prime}$ and $Y^{\prime}$ in Eq. (14) are extended to infinity, becoming a two-dimensional Fourier transform. On this basis, it results that

$$
\begin{aligned}
\left\langle I_{f}(U, V)\right\rangle= & \sum_{k, I=1}^{N} \mathfrak{F}\left\{\mid \mathfrak{F}\left\{P^{k l}(u, v)\right\}\right. \\
& \left.\times\left.\left(\frac{X+\Delta X^{k I}}{\lambda_{W} Z_{C}}, \frac{Y+\Delta Y^{k I}}{\lambda_{W} Z_{C}}\right)\right|^{2}\left(\frac{U}{\lambda_{R} f}, \frac{V}{\lambda_{R} f}\right)\right\} \\
= & \left(\lambda_{W} Z_{C}\right)^{2} \sum_{k, I=1}^{N} \exp \left[-i \frac{2 \pi}{\lambda_{R} f}\left(U \Delta X^{k l}\right.\right. \\
& \left.\left.+V \Delta Y^{k l}\right)\right] \\
& \times \mathfrak{F}\left\{\left.\mathfrak{F}\left\{P^{k l}(u, v)\right\}(X, Y)\right|^{2}\right\}(\vartheta U, \vartheta V), \quad(16)
\end{aligned}
$$

where $\vartheta=\left(\lambda_{W} Z_{C} / \lambda_{R} f\right)$. In what follows, the proportionality constant value $\left(\lambda_{W} Z_{C}\right)^{2}$ is omitted.

In Eq. (16) the summing terms for $k \neq 1$ can be separated from those that correspond to $k=1$. By taking into account that $P^{k l}=P^{l k}, P^{k k}=P^{k}$, and $\left(\Delta X^{k l}, \Delta Y^{k l}\right)$ $=-\left(\Delta X^{l k}, \Delta Y^{I k}\right)$, which implies that $\left(\Delta X^{k k}, \Delta Y^{k k}\right)=0$, from Eq. (16) it follows that

$$
\begin{aligned}
\left\langle I_{f}(U, V)\right\rangle= & \sum_{k=1}^{N} \mathfrak{F}\left\{\left|\mathfrak{F}\left\{P^{k}(u, v)\right\}(X, Y)\right|^{2}\right\}(\vartheta U, \vartheta V) \\
& +2 \sum_{\substack{k, I=1 \\
(k<I)}}^{N} \cos \left[\frac{2 \pi}{\lambda_{R} f}\left(U \Delta X^{k I}+V \Delta Y^{k l}\right)\right] \\
& \times \mathfrak{F}\left\{\left|\mathfrak{F}\left\{P^{k l}(u, v)\right\}(X, Y)\right|^{2}\right\}(\vartheta U, \vartheta V),
\end{aligned}
$$

where the smooth function

$\mathfrak{F}\left\{\left|\mathfrak{F}\left\{P^{k}(u, v)\right\}(X, Y)\right|^{2}\right\}(\vartheta U, \vartheta V)$

$$
\approx \int\left|P^{k}(\xi, \eta)\right|^{2}\left|P^{k}(\xi-\vartheta U, \eta-\vartheta V)\right|^{2} \mathrm{~d} \xi \mathrm{d} \eta
$$

represents the diffraction halo that corresponds to pupil $P^{k}{ }^{7.10}$ Then the first term in Eq. (17) describes the overlapping of all the smoothed diffraction halos, each one corresponding to an individual single-exposure recording $\mathfrak{F}\left\{\left|\mathfrak{F}\left\{P^{k}\right\}\right|^{2}\right\}$. Note that this term does not contribute to fringe formation. Similarly, the factor $\mathfrak{F}\left\{\left.t \mathfrak{F}\left\{P^{k h}\right\}\right|^{2}\right\}$ in the second term of Eq. (17) can be interpreted in terms of the halos associated with $P^{k l}$, which are fringe modulated. Note that each pair of pupils $P^{k}$ and $P^{l}$ can be associated with an independent contribution to fringe formation, related exclusively to the common part $P^{k l}$ of both pupils and the relative displacement of the respective images. Specifically, spatial frequencies that correspond to a relative displacement $\left(\Delta X^{k l}, \Delta Y^{k l}\right)$ are observed at the loci of the diffraction halo of $P^{k l}$. Nevertheless, if the common part of the pupils is null $\left[P^{k l}(u, v)=0\right]$, then no fringe formation can be associated with the $k$ th and the $l$ th recorded images. In summary, the second term of Eq. (17) stands for a rather complex pattern that results from the selective overlapping of elemental fringe-modulated diffraction spots. 
To illustrate the previous analysis we consider the particular case of a double-exposed specklegram $(N=2)$. As follows from Eq. (17),

$$
\begin{aligned}
\left\langle I_{f}(U, V)\right\rangle= & \mathfrak{F}\left\{\left|\mathfrak{F}\left\{P^{1}(u, v)\right\}(X, Y)\right|^{2}\right\}(\vartheta U, \vartheta V) \\
& +\mathfrak{F}\left\{\left|\mathfrak{F}\left\{P^{2}(u, v)\right\}(X, Y)\right|^{2}\right\}(\vartheta U, \vartheta V) \\
& +2 \cos \left[\frac{2 \pi}{\lambda_{R} f}\left(U \Delta X^{12}+V \Delta Y^{12}\right)\right] \\
& \times \mathfrak{F}\left\{\left|\mathfrak{F}\left\{P^{12}(u, v)\right\}(X, Y)\right|^{2}\right\}(\vartheta U, \vartheta V) .
\end{aligned}
$$

Observe that fringes are encountered only in those regions of the Fourier plane that coincide with the diffraction halo of the common part of the pupils. It follows that the common part of the pupils determines not only the correlation properties of the two speckled images, as relation (5b) has shown, but also the loci of fringes in the transform plane. In particular, fringes do not appear when two completely uncorrelated speckled images are recorded, that is, when the images are obtained through two pupils $P^{1}$ and $P^{2}$ for which $P^{12}=0$.

However, if the same pupil is employed to form both images in a double-exposed specklegram $\left(P^{1}=P^{2}\right.$ $\left.=P^{12}\right)$, then Eq. (19) becomes the well-known result, ${ }^{6-8}$

$$
\begin{aligned}
\left\langle I_{f}(U, V)\right\rangle= & 4 \cos ^{2}\left[\frac{\pi}{\lambda_{R} f}\left(U \Delta X^{12}+V \Delta Y^{12}\right)\right] \\
& \times \mathfrak{F}\left\{\left|\mathfrak{F}\left\{P^{1}(u, v)\right\}(X, Y)\right|^{2}\right\}(\vartheta U, \vartheta V),
\end{aligned}
$$

where $\mathfrak{F}\left\{\left|\mathfrak{F}\left\{P^{1}(u, v)\right\}(X, Y)\right|^{2}\right\}(\vartheta U, \vartheta V)$ gives the form of the respective diffraction halo [see relation (18)].

\section{Pupils with Common and Noncommon Apertures}

So far no restriction on the pupils to be employed has been imposed. Besides, it is important at this point to calculate the expressions for the amplitude and intensity cross-correlation functions and the average intensity in the transform plane for equal-aperture pupils that satisfy the conditions specified in the remainder of this subsection.

Each pupil $P^{k}(k=1,2, \ldots N)$ to be employed consists of an array of $q^{k}$ equal apertures represented by the functions $a_{n}^{k}(u, v)=a\left(u-u_{n}^{k}, v-v_{n}^{k}\right), n=1,2, \ldots q^{k}$. It is clear that $a_{n}^{k}(u, v)=1$ inside the $n$th aperture of the pupil $P^{k}$ and $a_{n}^{k}(u, v)=0$ otherwise. Because the loci of the aperture represented by $a_{n}^{k}(u, v)$ are determined by the point $\left(u_{n}^{k}, v_{n}^{k}\right)$, for simplicity we say that the aperture is located at $\left(u_{n}^{k}, v_{n}^{k}\right)$. Furthermore, there is no overlapping between two different apertures of the same pupil, that is, if $n \neq i$ then $a_{n}^{k}(u, v) a_{i}^{k}(u, v) \equiv 0$. Under these conditions, the pupil function is defined as $P^{k}(u, v)$ $=\sum_{n=1}^{q^{k}} a_{n}^{k}(u, v)$.

Also, it is assumed that, in a comparison of any pair of pupils $P^{k}$ and $P^{l}$, some of their apertures will coincide exactly, whereas other apertures will not overlap at all. Let the functions $a_{n}^{k}(u, v)$ and $a_{m}^{I}(u, v)$ represent two apertures that belong to two different pupils $(k \neq h)$, where $n=1,2, \ldots q^{k}$ and $m=1,2, \ldots q^{l}$. They are called common apertures if $a_{n}^{k}(u, v) \equiv a_{m}^{\prime}(u, v)$ and noncommon apertures if $a_{n}^{k}(u, v) a_{m}^{l}(u, v) \equiv 0$. Common and noncommon apertures can be identified whenever, for any possible pair of pupils $P^{k}$ and $P^{l}$, the pupil function $P^{k l}(u, v) \equiv P^{k}(u, v) P^{l}(u, v)$ results in a similar arrangement of $q^{k l}$ apertures represented by $a_{j}^{k l}(u, v)=a(u$ - $\left.u_{j}^{k l}, v-v_{j}^{k l}\right)$ for $j=1,2, \ldots q^{k l}\left(q^{k l} \leqslant q^{k}, q^{\prime}\right)$. Points $\left(u_{j}^{k l}, v_{j}^{k l}\right)$ are those that identify the positions of the common apertures. Finally, it can be written that $P^{k /}(u, v)$ $=\sum q_{j=1}^{k l} a_{j}^{k l}(u, v)$. Note that $P^{k k} \equiv P^{k}, q^{k k}=q^{k}$, and $\left(u_{j}^{k k}, v_{j}^{k k}\right)=\left(u_{j}^{k}, v_{j}^{k}\right)$.

\section{Evaluation of Cross-Correlation Functions}

For the pupils defined above and from relation (5b) and Eq. (15), the cross correlation for the amplitudes in the image plane is

$$
\begin{aligned}
\left\langle A^{k}\left(X_{a}, Y_{a}\right)\left[A^{l}\left(X_{b}, Y_{b}\right)\right]^{*}\right\rangle & \\
= & \left\{\sum_{j=1}^{q^{k l}} \exp \left[-i 2 \pi\left(u_{j}^{k l} \eta_{a b}^{k l}+v_{j}^{k l} \zeta_{a b}^{k l}\right)\right]\right\} \\
& \times \mathfrak{F}\{a(u, v)\}\left(\eta_{a b}^{k l}, \zeta_{a b}^{k l}\right),
\end{aligned}
$$

where

$$
\begin{aligned}
\left(\eta_{a b}^{k l}, \zeta_{a b}^{k l}\right) \equiv & \left(1 / \lambda_{w} Z_{C}\right)\left(X_{a}-X_{b}-\Delta X^{k l}, Y_{a}-Y_{b}\right. \\
& \left.-\Delta Y^{k l}\right) .
\end{aligned}
$$

From Eq. (21) it follows that

$$
\left\langle I^{k}\right\rangle=q^{k} \mathfrak{F}\left\{a^{k}(u, v)\right\}(0,0)
$$

and

$$
\begin{aligned}
\left|\left\langle A^{k}\left(X_{a}, Y_{a}\right)\left[A^{I}\left(X_{b}, Y_{b}\right)\right]^{*}\right\rangle\right|^{2} & \left(q^{k I}+2 \sum_{\substack{i, j=1 \\
(i<j)}}^{q^{k I}} \cos \left\{2 \pi \left[\left(u_{i}^{k I}-u_{j}^{k I}\right) \eta_{a b}^{k l}\right.\right.\right. \\
& \left.\left.\left.+\left(v_{i}^{k I}-v_{j}^{k l}\right) \zeta_{a b}^{k I}\right]\right\}\right)\left|\mathfrak{F}\{a(u, v)\}\left(\eta_{a b}^{k l}, \zeta_{a b}^{k I}\right)\right|^{2} .
\end{aligned}
$$

Then, from Eqs. (7), (22), and (23), the cross correlation of intensities is

$$
\begin{aligned}
\left\langle I^{k}\left(X_{a}, Y_{a}\right) I^{I}\left(X_{b}, Y_{b}\right)\right\rangle & \\
= & q^{k} q^{l}[\mathfrak{F}\{a(u, v)\}(0,0)]^{2} \\
& +\left(q^{k I}+2 \sum_{\substack{i, j=1 \\
(j<j)}}^{q^{k I}} \cos \left\{2 \pi \left[\left(u_{i}^{k I}-u_{j}^{k I}\right) \eta_{a b}^{k I}\right.\right.\right. \\
& \left.\left.\left.+\left(v_{i}^{k I}-v_{j}^{k I}\right) \zeta_{a b}^{k I}\right]\right\}\right)\left|\mathfrak{F}\{a(u, v)\}\left(\eta_{a b}^{k I}, \zeta_{a b}^{k l}\right)\right|^{2} .
\end{aligned}
$$

\section{Evaluation of Average Intensity}

If we consider that $N$ images are recorded by use of a set of pupils that satisfy the conditions $P^{k}(u, v)$ $=\sum_{n=1}^{q^{k}} a_{n}^{k}(u, v)$ and $P^{k l}(u, v)=\sum_{j=1}^{q^{k l}} a_{j}^{k l}(u, v)$, where the function $a(u, v)$ defines the aperture shape, it follows that 
$\mathfrak{F}\left\{\left|\mathfrak{F}\left\{P^{k}(u, v)\right\}(X, Y)\right|^{2}\right\}(\vartheta U, \vartheta V)$

$$
\begin{aligned}
= & \mathfrak{F}\left\{\left|\mathfrak{F}\left\{\sum_{j=1}^{q^{k}} a\left(u-u_{j}^{k}, v-v_{j}^{k}\right)\right\}(X, Y)\right|^{2}\right\}(\vartheta U, \vartheta V) \\
= & \mathfrak{F}\left\{\left|\mathfrak{F}\{a(u, v)\}(X, Y) \sum_{j=1}^{q^{k}} \exp \left[-i 2 \pi\left(X u_{j}^{k}+Y V_{j}^{k}\right)\right]\right|^{2}\right\} \\
& \times(\vartheta U, \vartheta V) \\
= & \mathfrak{F}\left\{|\mathfrak{F}\{a(u, v)\}(X, Y)|^{2}\right. \\
& \times\left(q^{k}+2 \sum_{\substack{r, s=1 \\
(r<s)}}^{q^{k}} \cos \left\{2 \pi \left[X\left(u_{r}^{k}-u_{s}^{k}\right)\right.\right.\right. \\
& \left.\left.\left.\left.+Y\left(v_{r}^{k}-v_{s}^{k}\right)\right]\right\}\right)\right\}(\vartheta U, \vartheta V) .
\end{aligned}
$$

and similarly

$\mathfrak{F}\left\{\left|\mathfrak{F}\left\{P^{k l}(u, v)\right\}(X, Y)\right|^{2}\right\}(\vartheta U, \vartheta V)$

$$
\begin{aligned}
= & \mathfrak{F}\left\{| \mathfrak { F } \{ a ( u , v ) \} ( X , Y ) | ^ { 2 } \left(q^{k I}+2 \sum_{\substack{r, s=1 \\
(r<s)}}^{q^{k}} \cos \left\{2 \pi \left[X \left(u_{r}^{k I}\right.\right.\right.\right.\right. \\
& \left.\left.\left.\left.\left.-u_{s}^{k l}\right)+Y\left(v_{r}^{k I}-v_{s}^{k I}\right)\right]\right\}\right)\right\}(\vartheta U, \vartheta V) .
\end{aligned}
$$

By substituting Eqs. (25) into Eq. (17) and rearranging the summing terms, we obtain finally, the average intensity,

$$
\left\langle I_{f}(U, V)\right\rangle=\left\langle I_{f}^{0}(U, V)\right\rangle+\left\langle I_{f}^{L}(U, V)\right\rangle,
$$

where

$\left\langle I_{r}^{0}(U, V)\right\rangle$

$$
=\left\{\sum_{k=1}^{N} q^{k}+2 \sum_{\substack{k, J=1 \\(k<l)}}^{N} q^{k l} \cos \left[\frac{2 \pi}{\lambda_{R} f}\left(U \Delta X^{k l}+V \Delta Y^{k l}\right)\right]\right\}
$$$$
\times \mathfrak{F}\left\{|\mathfrak{F}\{a(u, v)\}(X, Y)|^{2}\right\}(\vartheta U, \vartheta V),
$$

$\left\langle I_{f}^{L}(U, V)\right\rangle$

$$
\begin{aligned}
= & \sum_{k=1}^{N} \sum_{\substack{r, s=1 \\
(r \neq s)}}^{q^{k}} \mathfrak{F}\left\{|\mathfrak{F}\{a(u, v)\}(X, Y)|^{2}\right\} \\
& \times\left(\vartheta U+u_{r}^{k}-u_{s}^{k}, \vartheta V+v_{r}^{k}-v_{s}^{k}\right) \\
& +2 \sum_{\substack{k, l=1 \\
(k<l)}}^{N} \cos \left[\frac{2 \pi}{\lambda_{R} f}\left(U \Delta X^{k l}+V \Delta Y^{k I}\right)\right] \\
& \times \sum_{\substack{r, s=1 \\
(r \neq s)}}^{q^{k l}} \mathfrak{F}\left\{|\mathfrak{F}\{a(u, v)\}(X, Y)|^{2}\right\} \\
& \times\left(\vartheta U+u_{r}^{k l}-u_{s}^{k l}, \vartheta V+v_{r}^{k l}-v_{s}^{k l}\right) .
\end{aligned}
$$

By defining the function

$$
\mathcal{\kappa}(U, V) \equiv \mathfrak{F}\left\{|\mathfrak{F}\{a(u, v)\}(X, Y)|^{2}\right\}(\vartheta U, \vartheta V)
$$

it is possible to write the average intensities given by Eqs. (27) and (28) as follows:

$$
\begin{aligned}
& \left\langle I_{f}^{0}(U, V)\right\rangle=\left\{\sum_{k=1}^{N} q^{k}+2 \sum_{\substack{k, I=1 \\
(k<1)}}^{N} q^{k l} \cos \left[\frac { 2 \pi } { \lambda _ { R } f } \left(U \Delta X^{k I}\right.\right.\right. \\
& \left.\left.\left.+V \Delta Y^{k l}\right)\right]\right\} \kappa(U, V) \\
& \left\langle I_{f}^{L}(U, V)\right\rangle=\sum_{k=1}^{N} \sum_{\substack{r, s=1 \\
(r \neq s)}}^{q^{k}} \kappa\left(U-U_{r s}^{k}, V-V_{r s}^{k}\right) \\
& +2 \sum_{\substack{k, l=1 \\
(k<l)}}^{N} \cos \left[\frac{2 \pi}{\lambda_{R} f}\left(U \Delta X^{k l}+V \Delta Y^{k l}\right)\right] \\
& \times \sum_{\substack{r, s=1 \\
(r \neq s)}}^{q^{k l}} \kappa\left(U-U_{r s}^{k l}, V-V_{r s}^{k l}\right)
\end{aligned}
$$

where

$$
\begin{aligned}
& \left(U_{r s}^{k}, V_{r s}^{k}\right) \equiv\left(\lambda_{R} f\left(\lambda_{W} Z_{C}\right)\left(u_{s}^{k}-u_{r}^{k}, v_{s}^{k}-v_{r}^{k}\right),\right. \\
& \left(U_{r s}^{k l}, V_{r s}^{k l}\right) \equiv\left(\lambda_{R} f \lambda_{W} Z_{C}\right)\left(u_{s}^{k I}-u_{r}^{k l}, v_{s}^{k l}-v_{r}^{k l}\right) .
\end{aligned}
$$

The function $\aleph(U, V)$ is proportional to the ensembleaverage intensity distribution of the diffraction halo for a single-exposed speckle pattern recorded through a pupil consisting of only one of the apertures.

For example, if the pupil consists of circular apertures of diameter $D$, then

$$
\begin{aligned}
\kappa(U, V)= & \frac{D^{2}}{2}\left\{\cos ^{-1}\left(\frac{2 \rho}{D^{\prime}}\right)-\left(\frac{2 \rho}{D^{\prime}}\right)\left[1-\left(\frac{2 \rho}{D^{\prime}}\right)^{2}\right]^{1 / 2}\right\} \\
& \times \operatorname{cyl}\left(\frac{\rho}{D^{\prime}}\right)
\end{aligned}
$$

where

$$
\begin{gathered}
\rho=\sqrt{U^{2}+V^{2},} \quad D^{\prime}=\left(2 f / Z_{C}\right)\left(\lambda_{R} / \lambda_{W}\right) D, \\
\operatorname{cyl}\left(\rho / D^{\prime}\right)= \begin{cases}1 & \text { if } 0 \leqslant \rho \leqslant D^{\prime} / 2 \\
0 & \text { otherwise }\end{cases}
\end{gathered}
$$

In this case $\aleph(U, V)$ stands for a (smoothed) circular diffraction spot of diameter $D^{\prime}$, centered at the origin of the $U-V$ plane. In addition, the function $\aleph(U-\alpha, V-\beta)$ represents a spot that is similar but centered at the point $(\alpha, \beta)$.

\section{Diffraction Patterns with Common and Noncommon Spots \\ In speckle photography applications through multiple- aperture pupils, some advantage can be had if separated diffraction spots are observed in the Fourier plane, for ex- ample, for avoidance of cross-talk effects in a variety of image multiplexing approaches and for noise reduction by}




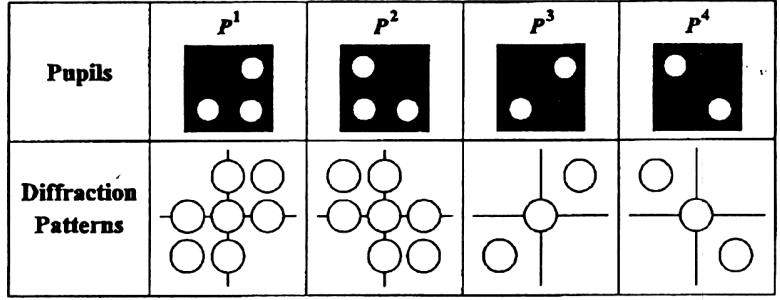

Fig. 2. Diffraction pattern schemes corresponding to the four pupils employed.

spatial filtering in the transform plane. This condition can be fulfilled by proper design of the pupils, as is assumed in the following.

It is convenient to impose another condition: Let us assume that comparison of the two diffraction patterns corresponding to any pair of the pupils to be employed shows that the loci of some spots of one diffraction pattern coincide exactly with some diffraction spots of the other pattern, while the remaining spots do not overlap at all. To illustrate this point, in the first row of Fig. 2 four pupils are schematized, meanwhile in the second row the respective diffraction patterns are depicted. By observing the diffraction patterns that belong to pupils $P^{1}$ and $P^{2}$, we can see that the spots located along the horizontal and vertical axes are common spots, whereas the lateral spots centered on the diagonals are not shared and are therefore called noncommon spots. Of course, the zero order is always a common diffraction spot, irrespective of the pattern observed. In the same sense in which the common and noncommon apertures were defined for the pupils, in this context it is assumed that common and noncommon spots can be identified when any pair of diffraction patterns is observed for the pupils.

In summary, the shapes of all the apertures of the pupils are identical; for any pair of pupils, common and noncommon apertures can be associated with them; the diffraction pattern corresponding to each pupil consists of separated spots; and common and noncommon diffraction spots can be associated with any pair of the diffraction patterns that the pupils individually generate.

The advantage of using different multiple-aperture pupils that satisfy these working conditions is that we can thus selectively isolate or combine the spectral information of several multiplexed images in separate zones of the diffraction plane. ${ }^{11}$ For example, in the case of a double exposure through pupils $P^{1}$ and $P^{2}$ of Fig. 2, the spectral amplitude components for both exposures are added into the common diffraction spots, while in the noncommon spots their individual spectra are isolated.

\section{Features of the Diffracted Spots}

In this subsection the average expressions $\left\langle I_{f}^{0}(U, V)\right\rangle$ and $\left\langle I_{f}^{L}(U, V\rangle\right.$ in Eqs. (30) and (31) are interpreted on the basis of the detailed working conditions. Also, the average intensity of the diffraction spots is analytically obtained and the fringe visibility is analyzed.

As a starting point, note that the location of the spot $\kappa(U-\alpha, V-\beta)$ is unambiguously determined by the point $(\alpha, \beta)$, and, in this sense, we say that this spot is located at $(\alpha, \beta)$. Moreover, the spots represented by $\kappa(U$ $-\alpha, V-\beta)$ and $\kappa(U-\sigma, V-\omega)$ coincide only if $\alpha$ $=\sigma$ and $\beta=\omega$ [see Eq. (29)].

Observe that the average intensity $\left\langle I_{f}^{L}(U, V)\right\rangle$ results from the overlapping of all those spots represented by $\kappa(U-\alpha, V-\beta)$ with $(\alpha, \beta) \neq 0$. This is because the inequality $r \neq-s$ implies both that $\left(U_{r s}^{k}, V_{r s}^{k}\right) \neq 0$ and that $\left(U_{r s}^{k I}, V_{r s}^{k l}\right) \neq 0$. Furthermore, for each term of the form $N(U-\alpha, V-\beta)$ in Eq. (31) there is always another term of the form $\kappa(U+\alpha, V+\beta)$ because $\left(U_{r s}^{k}, V_{r s}^{k}\right)$ $=-\left(U_{s r}^{k}, V_{s r}^{k}\right)$ and $\left(U_{r s}^{k l}, V_{r s}^{k l}\right)=-\left(U_{s r}^{k l}, V_{s r}^{k l}\right)$. Then the intensity $\left\langle I_{f}^{L}(U, V)\right\rangle$ results from the overlapping of those spot pairs that are symmetrically located with respect to the origin of the $U-V$ plane, whereas all the terms in Eq. (30) are located at $(\alpha, \beta)=0$. It can be concluded that $\left\langle I_{f}^{L}(U, V)\right\rangle$ represents the average intensity distribution for the lateral diffraction spots and that $\left\langle I_{f}^{0}(U, V)\right\rangle$ represents the zero-order contribution in the Fourier plane.

Let us define the numbers $q_{\alpha \beta}^{k}$ and $q_{\alpha \beta}^{k I}$ as follows: For a given spot located at $(\alpha, \beta) \neq 0, q_{\alpha \beta}^{k}$ is the number of aperture pairs in the pupil $P^{k}$ for which $\left(u_{s}^{k}-u_{r}^{k}, v_{s}^{k}\right.$ - $\left.v_{r}^{h}\right)=\left(\lambda_{W} Z_{C} / \lambda_{R} f\right)(\alpha, \beta)$ or, equivalently, $\left(U_{r s}^{k}, V_{r s}^{k}\right)$ $=(\alpha, \beta)$. Similarly, for $(\alpha, \beta) \neq 0, q_{\alpha \beta}^{k l}$ is the number of common aperture pairs in the pupil $P^{k l}$ for which $\left(U_{r s}^{k l}, V_{r s}^{k l}\right)=(\alpha, \beta)$. Also, for $(\alpha, \beta)=0, q_{\alpha \beta}^{k}$ and $q_{\alpha \beta}^{k l}$ are the numbers of apertures in the pupils $P^{k}$ and $P^{k l}$, respectively.

On the basis of these definitions, Eqs. (26), (30), and (31) are written in a compact form that exhibits the main features of speckle photography through different multiple-aperture pupils but is easier to interpret and more useful in practical situations. After rearranging terms, we find the average intensity of the diffraction spot located at $(\alpha, \beta)$ in the Fourier plane:

$$
\begin{aligned}
\left\langle I_{\alpha \beta}(U, V)\right\rangle & \\
= & \left\{\sum_{k=1}^{N} q_{\alpha \beta}^{k}+2 \sum_{\substack{k, I=1 \\
(k<I)}}^{N} q_{\alpha \beta}^{k l} \cos \left[\frac{2 \pi}{\lambda_{R} f}\left(U \Delta X^{k l}+V \Delta Y^{k l}\right)\right]\right\} \\
& \times \kappa(U-\alpha, V-\beta) .
\end{aligned}
$$

The first term of Eq. (33) is not associated with fringe formation in the spot at the position $(\alpha, \beta)$. The number $q_{\alpha \beta}^{k I}$ in the second term is a weighting factor that determines the magnitude of the contribution of the pupil $P^{k l}$ to fringe formation. However, only for the zero order are the numbers $q_{\alpha \beta}^{k}$ and $q_{\alpha \beta}^{k I}$ proportional to the aperture transmission areas of the pupils $P^{k}$ and $P^{k I}$, respectively.

For simplicity, let us consider the case of a doubleexposed specklegram. Equation (33) becomes

$$
\begin{aligned}
\left\langle I_{\alpha \beta}(U, V)\right\rangle & \\
= & \left\{q_{\alpha \beta}^{1}+q_{\alpha \beta}^{2}+2 q_{\alpha \beta}^{12} \cos \left[\frac{2 \pi}{\lambda_{R} f}\left(U \Delta X^{12}+V \Delta Y^{12}\right)\right]\right\} \\
& \times \kappa(U-\alpha, V-\beta) .
\end{aligned}
$$

In each recording step, a fringe-modulated speckle pattern is stored. Although a rather complex system of fringes modulates the speckles, it can be understood in terms of the overlapping of elemental fringe systems. ${ }^{9.11}$ All those aperture pairs in the pupil $P^{k}(k=1,2)$ for which $\left(u_{r}^{k}-u_{s}^{k}, v_{r}^{k}-y_{s}^{h}\right)=\left(\lambda_{w} Z_{C} / \lambda_{R} f\right)(\alpha, \beta)$ stand for 
an elemental fringe system into the imaged speckles, whose mean spatial period is $p_{\alpha \beta}^{k}=\lambda_{R} f f\left(\alpha^{2}+\beta^{2}\right)^{1 / 2}$ and whose orientation is determined by the angle $\theta_{\alpha \beta}^{k}$ $=\tan ^{-1}(\beta / \alpha)$. In the analysis step, light diffracted by this fringe system goes to the spot located at $(\alpha, \beta)$ in the $U-V$ plane. Then the numbers $q_{\alpha \beta}^{1}$ and $q_{\alpha \beta}^{2}$ in Eq. (34) also represent the number of aperture pairs in the respective pupils $P^{1}$ and $P^{2}$ that are responsible for a spot formation at $(\alpha, \beta)$.

In the analysis step of the specklegram, light is diffracted by the speckles that were recorded in both the first and the second exposure. The shape and relative position of the apertures in the pupils control the spectral content of light spread out by the diffuser that is recorded. Only if both numbers $q_{\alpha \beta}^{1}$ and $q_{\alpha \beta}^{2}$ are not zero do the spectral components of both images overlap in the spot at point $(\alpha, \beta)$. Nevertheless, for fringe formation it is not enough that an overlapping of the spectral content of both images occurs, because fringes appear only in the loci of the diffraction halo that corresponds to the common part of the two pupils, where $q_{\alpha \beta}^{12} \neq 0$. In fact, the overlapping of spectral components into a given spot depends on the internal modulation of the imaged speckles, irrespective the correlation properties of the recorded speckle distributions. However, correlation between modulated speckles that diffract light into a given spot depends on the number of common aperture pairs in the pupils that contribute to their internal modulation. Furthermore, for a given diffraction spot, parts of the spectral information accepted by the pupils coincide if there are common aperture pairs in the pupils associated with that spot formation. However, when no such common apertures exist, the spectral components that correspond to different recordings are not related to each other. When different multiple-aperture pupils are employed, it is possible that the spectra associated with common and noncommon apertures are concentrated in a determined spot. In this case, the information that corresponds to noncommon apertures does not contribute to fringe formation, thus reducing the visibility of the correlation fringes. In consequence, a judicious selection of the pupils is most important to ensure that the spectral information in different spots corresponds solely to diffracted light associated with the aperture pairs that the pupils have in common.

Figure 3 depicts the average intensity profile given by Eq. (34) along an axis that is parallel to the $U$ axis and passes through the spot centered at $(\alpha, \beta)$. For plotting, circular apertures were considered [see Eq. (32)].

Fringe visibility is defined as $s^{6,12}$

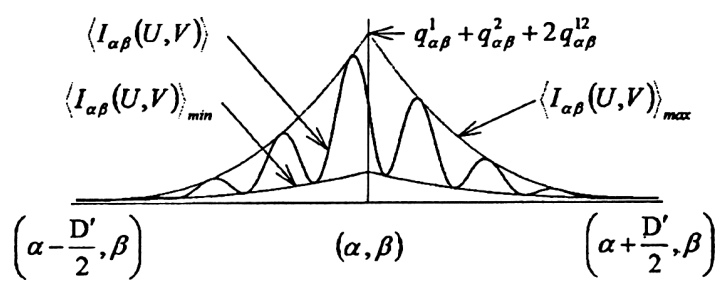

Fig. 3. Average intensity profile for a spot centered at $(\alpha, \beta)$ in the $U-V$ plane.

$$
\mathcal{V}_{\alpha \beta}=\frac{\left\langle I_{\alpha \beta}(U, V)\right\rangle_{\max }-\left\langle I_{\alpha \beta}(U, V)\right\rangle_{\min }}{\left\langle I_{\alpha \beta}(U, V)\right\rangle_{\max }+\left\langle I_{\alpha \beta}(U, V)\right\rangle_{\min }},
$$

where the intensities $\left\langle I_{\alpha \beta}(U, V)\right\rangle_{\max }$ and $\left\langle I_{\alpha \beta}(U, V)\right\rangle_{\min }$ are the envelopes (also plotted in Fig. 2) given by

$$
\begin{aligned}
& \left\langle I_{\alpha \beta}(U, V)\right\rangle_{\max }=\left\{q_{\alpha \beta}^{1}+q_{\alpha \beta}^{2}+2 q_{\alpha \beta}^{12}\right\} \aleph(U-\alpha, V-\beta), \\
& \left\langle I_{\alpha \beta}(U, V)\right\rangle_{\min }=\left\{q_{\alpha \beta}^{1}+q_{\alpha \beta}^{2}-2 q_{\alpha \beta}^{12}\right\} \kappa(U-\alpha, V-\beta) .
\end{aligned}
$$

Then, according to Eqs. (36), the visibility is

$$
\mathcal{V}_{\alpha \beta}=2 \frac{q_{\alpha \beta}^{12}}{q_{\alpha \beta}^{1}+q_{\alpha \beta}^{2}} .
$$

Note that, as is well known, $0 \leqslant \mathcal{V}_{\alpha \beta} \leqslant 1$ because $q_{\alpha \beta}^{12}$ $\leqslant q_{\alpha \beta}^{1}, q_{\alpha \beta}^{2}$. The visibility is null $\left(\mathcal{V}_{\alpha \beta}=0\right)$ at the point $(\alpha, \beta)$ whenever $q_{\alpha \beta}^{12}=0$. In particular, if the pupils have no common apertures $\left[P^{12}(u, v)=0\right]$, then no correlation exists and the visibility fades out. However, if the pupils coincide, then $P^{12}(u, v)=P^{1}(u, v)=P^{2}(u, v)$ and $q_{\alpha \beta}^{12}=q_{\alpha \beta}^{1}=q_{\alpha \beta}^{2}$. As expected, in this case Eq. (34) reduces to Eq. (20), and the predicted visibility reaches unity for all the spots. In general, we have partially correlated speckle images, and visibility values are less than 1. In particular, if $q_{\alpha \beta}^{1}=q_{\alpha \beta}^{2}$, then $\mathcal{V}_{\alpha \beta}=q_{\alpha \beta}^{12}$.

\section{EXPERIMENTAL RESULTS}

In this section the validity of Eqs. (33), (34), and (37) is analyzed. The procedures implemented are detailed in the following.

\section{A. Double-Exposed Specklegrams}

We recorded each imaged speckle distribution by using the conventional experimental arrangement shown in Fig. 1(a). A diffuser was illuminated by a collimated Arlaser beam $\left(\lambda_{W}=514 \mathrm{~nm}\right)$ and imaged onto the $X-Y$ plane. In this plane a CCD TV camera was located to capture and then to digitize the image. The distances from the diffuser to the lens and from the lens to the camera were set to $Z_{0}=138 \mathrm{~mm}$ and $Z_{\mathrm{C}}=382 \mathrm{~mm}$, respectively. Pupil masks with circular holes of diameter $D$ $=4.8 \mathrm{~mm}$ and centered at the vertices of a square $d$ $=9.2 \mathrm{~mm}$ on a side were used. The digitized images were multiplexed to simulate the in-plane displacement between them. Also, the process of analysis was digitally simulated by use of a fast-Fourier-transform (FFT) algorithm.

Each row in Figs. 4-6 corresponds to a double-exposed specklegram obtained by use of the pupils shown schematically in the first column. In each case, for the second exposure an actual uniform horizontal in-plane displacement of the diffuser $(40 \mu \mathrm{m})$ was simulated. The displacement in the image plane $(\approx 25 \mu \mathrm{m})$ is larger than the average image speckle size $(\approx 50 \mu \mathrm{m})$. Because no structural change of speckle patterns attributable to displacement is introduced, decorrelation is due only to the pupil change. A conventional FFT algorithm was employed to simulate the analog analysis process presented in Fig. 1(b). From the FFT data, the modulus of the Fourier transform $\left[I_{f}(U, V)\right]^{1 / 2}$ is calculated. Each image in 
the second column in Figs. 4-6 represents the $\ln \{1$ $+\left[I_{f}(U, V]^{1 / 2}\right\}$ data. Also, the thick profiles of the third column correspond to the horizontal upper and central spots of the respective low-pass-filtered $\left[I_{f}(U, V)\right]^{1 / 2} \mathrm{im}$ ages. For all the thick profiles in Figs. 4-6 the maximum

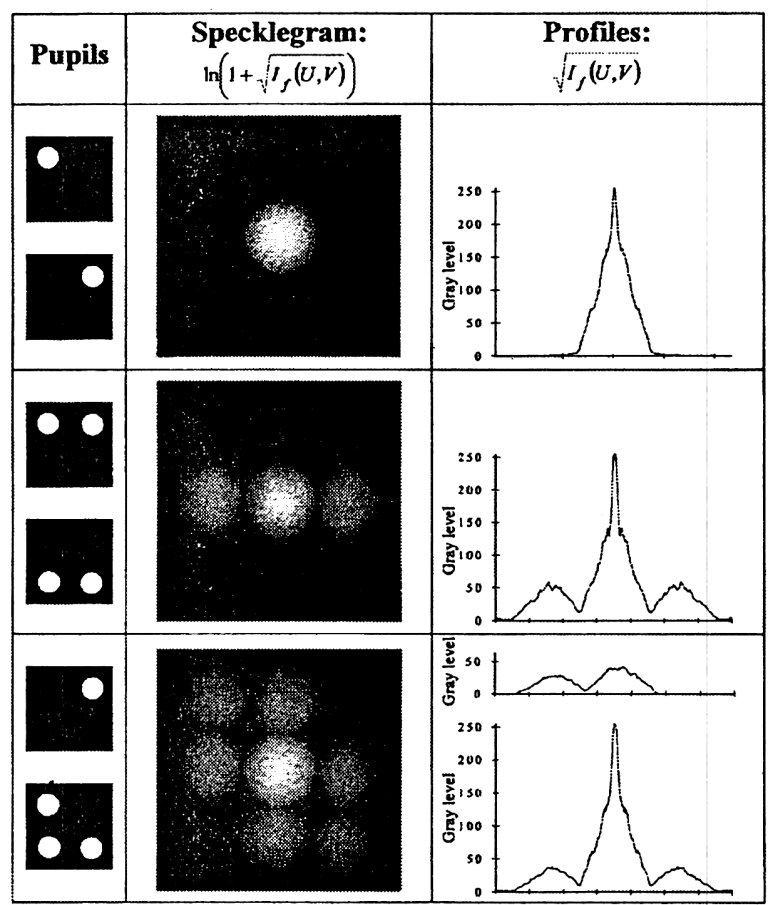

Fig. 4. Double-exposed specklegrams through pupils without common apertures.

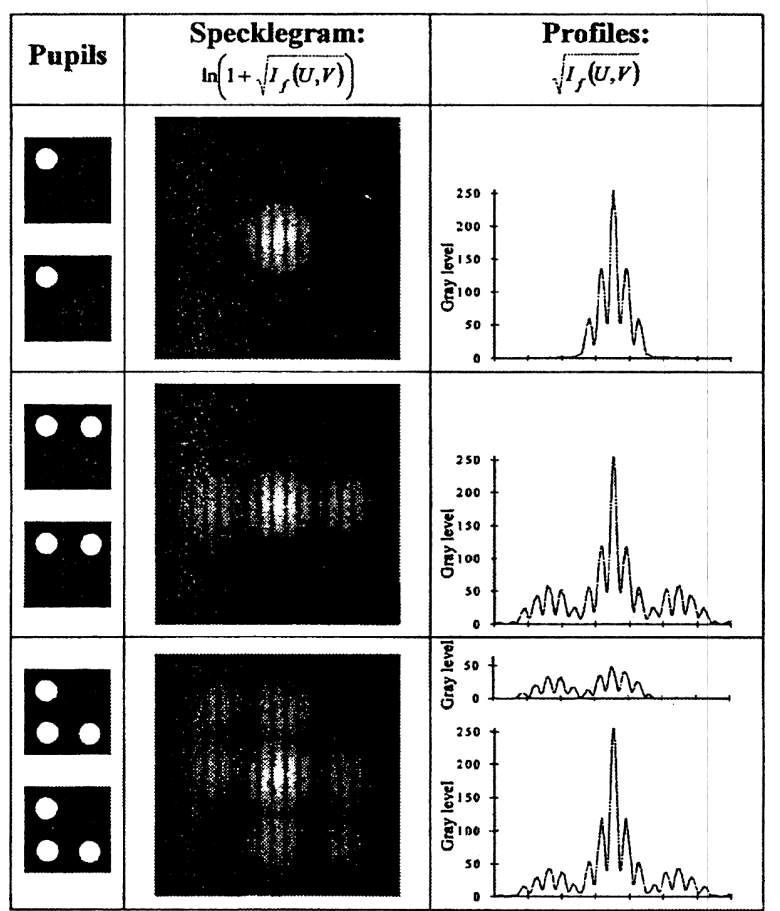

Fig. 5. Double-exposed specklegrams through identical pupils.

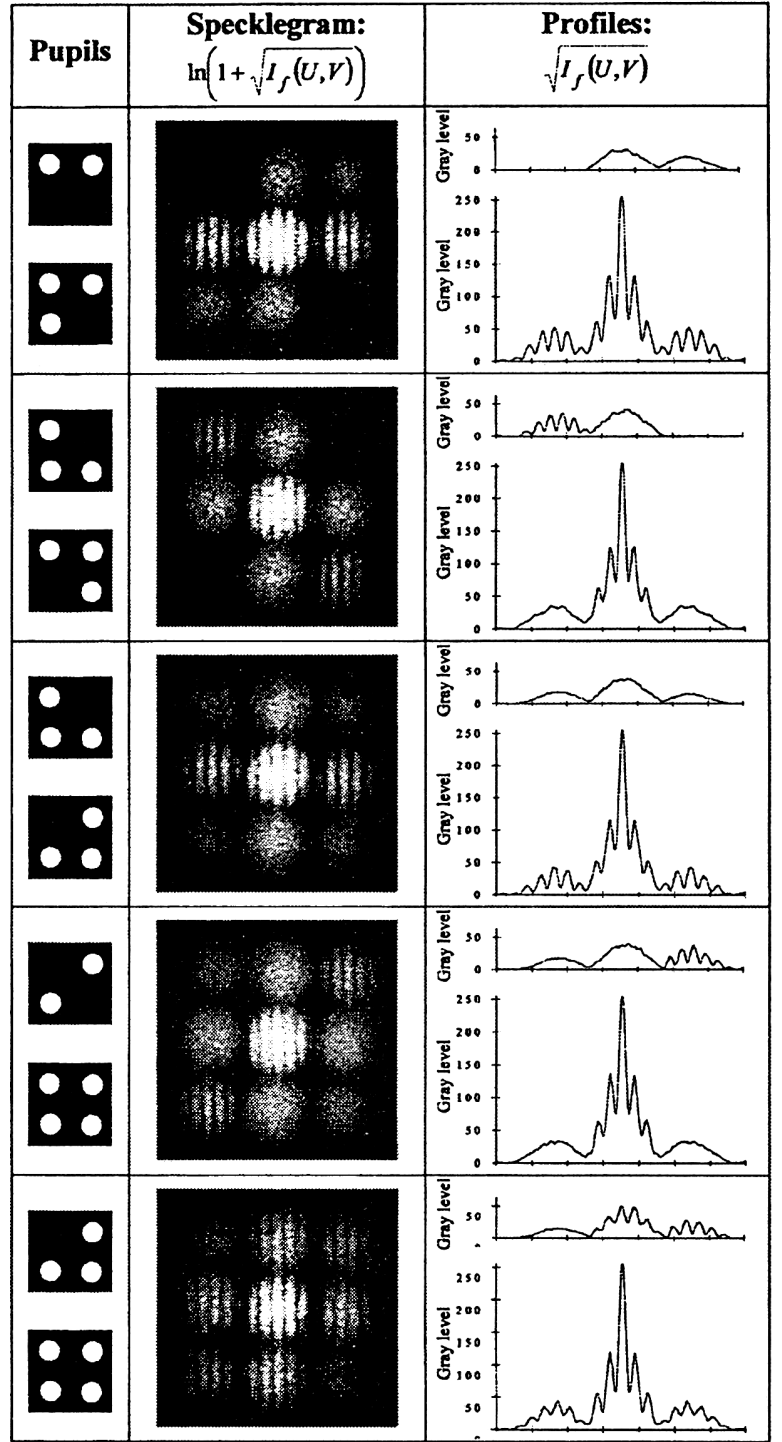

Fig. 6. Double-exposed specklegrams through pupils with common and noncommon apertures.

value was set to the same gray level. This implies that the peak values of lateral spots are diminished in proportion to the absolute peak values of the corresponding central spots. Note that smoothed profiles are obtained because of the averaging effect of both the low-pass-filtering operation and the selection of a rather thick region for sampling. This effect is equivalent to statistical averaging over an ensemble of diffusers. Circular spots are observed in the Fourier plane, as expected, when pupils with circular apertures are used [see Eq. (32)].

The results of Fig. 4 confirm that no fringes are observed $\left(\mathcal{V}_{\alpha \beta}=0\right)$ in the transform plane when the two recorded speckle patterns are completely uncorrelated: $\left\langle A^{1}\left(A^{2}\right)^{*}\right\rangle=0$. This is so because the two pupils in each row of this figure have no common apertures $\left(P^{12} \equiv 0\right)$.

Figure 5, however, shows some typical results for uniform in-plane displacements in speckle photography. ${ }^{8}$ The optimum fringe visibility for all the spots is observed 
because in this case the two pupils coincide $\left(P^{1}=P^{2}\right.$ $=P^{12}$ ) and in consequence the speckle patterns are fully correlated. Observe that, although the theoretically predicted visibility equals unity (irrespective of which spot is of concern), the actual experiments show values that are always less than unity. This result is a consequence of several effects, and some of them are as follows. In practice, the recording procedure is not linear ${ }^{7}$; also, electronic noise is of concern, ${ }^{14}$ mainly because of the high dynamic range of the intensity distribution of a Gaussian speckle, whose most probable value is zero. Also, an integrated (or smoothed) speckle pattern is recorded because of the finite area of the detector array in the CCD camera. ${ }^{10}$ However, when a speckle pattern is digitally displaced, a portion of the original pattern goes out of and another portion enters into the new frame, as occurs when an actual object displacement takes place between expositions. In consequence, after the two speckle patterns are added, the number of pairs of corresponding speckles within the image frame decreases as the displacement increases. This effect cannot be eliminated and stands for a reduction of interferometric fringe visibility. ${ }^{6,12}$ Furthermore, some drawbacks are related to finite areas of integration and digital operations, such as local saturation when speckle patterns and approximations of the FFT algorithm are added.

In Fig. 6 some results obtained by use of two different multiple-aperture pupils are illustrated. In each case, isolated common and noncommon diffraction spots can be identified by comparison of the respective diffraction patterns. Points $(0,0), \pm\left(d^{\prime \prime}, 0\right), \pm\left(0, d^{\prime \prime}\right), \pm\left(d^{\prime}, d^{\prime}\right)$, and $\pm\left(d^{\prime},-d^{\prime}\right)$, where $d^{\prime}=d\left(\lambda_{R} f / \lambda_{w} Z_{C}\right)$, denote the generic positions for the spot centers. For simplicity, let us define $d^{+}=-d^{-}=d^{\prime \prime}$. In the following, only one spot for each pair of lateral spots is considered, because two spots located symmetrically with respect to the origin have the same characteristics.

As an example, let us analyze in detail the specklegram depicted in the fourth row of Fig. 6. Light into the spot at $\left(d^{+}, 0\right)$ carries only the spectral content of the image recorded with the four-aperture pupil $\left(q_{d^{+}}^{1}=-0\right.$ and $q_{d^{+} 0}^{2} \equiv-2$ ), and no fringes are observed because the pupils have no common aperture pairs responsible for that spot formation $\left(q_{d^{+} 0}^{12}=0\right.$, then $\left.\mathcal{V}_{d^{+} 0}=-0\right)$. The spot located at $\left(0, d^{+}\right)$exhibits the same features $\left(q_{0 d^{+}}^{1}=q_{0 d^{+}}^{12}=0\right.$, $q_{0 d^{+}}^{2} \equiv 2$ and $\left.\mathcal{V}_{0 d^{+}} \equiv-0\right)$ as in the previous case, which is apparent when one compares the respective thick profiles for the spots at $\left(d^{+}, 0\right)$ and $\left(0, d^{+}\right)$. Moreover, for the spot at $\left(d^{-}, d^{+}\right)$, similar conclusions can be established, in that no fringes are observed $\left(q_{d^{-} d^{+}}^{1}=q_{d^{-} d^{+}}^{12}=0\right.$ and $\left.q_{d^{\prime} d^{+}}^{2}=1\right)$. Besides, the average intensity in this spot is lower (one half) than in the spots at $\left(d^{+}, 0\right)$ and $\left(0, d^{+}\right)$, as one can observe in the corresponding thick profiles. For the spot at $\left(d^{+}, d^{+}\right)$, the spectral content of both the first and the second images are overlapped $\left(q_{d^{+} d^{+}}^{1}\right.$ $\left.=q_{d^{+} d^{+}}^{2}=1\right)$. Furthermore, fringes are observed there because the common part of the pupils includes one pair of apertures associated with this spot formation $\left(q_{d^{+} d^{+}}^{12}\right.$ $\equiv 1$ ). As results when the same pupils are used for recording, for this spot it holds that $\left\langle I_{d^{+} d^{+}}(U, V)\right\rangle$ $=4 \cos ^{2}\left(\pi U \Delta X^{12} / \lambda_{R} f\right) \wedge\left(U-d^{+}, V=d^{+}\right)$, and the pre- dicted visibility is unity. Thick profiles show that fringe modulations for this spot and for the lateral spots in Fig. 5 are comparable. In the zero order the spectra of both images are overlapped $\left(q_{00}^{1}=2\right.$ and $\left.q_{00}^{2}=4\right)$, and fringes are observed because the pupils have two apertures in common $\left(q_{00}^{12}=2\right)$. Besides, $\mathcal{V}_{00}=2 / 3$ because not all the apertures are shared by the pupils. In this case $\left\langle I_{00}(U, V)\right\rangle=\left\{6+4 \cos \left(2 \pi U \Delta X^{12} / \lambda_{R} f\right)\right\} \kappa(U, V)$.

In the specklegram of the fifth row in Fig. 6 , more than one pair of lateral diffraction spots are fringe modulated. Also, only the spots located at $\left(d^{+}, d^{+}\right)$and $\left(d^{\bar{\sim}}, d^{-}\right)$exhibit optimum visibility. For the spots at $\left(0, \pm d^{+}\right)$and $\left( \pm d^{+}, 0\right)$ the predicted visibility is $2 / 3$; for the zero order it is 6/7. From a comparison of the thick profiles for the spots at $\left(d^{+}, d^{+}\right)$and $\left(0, d^{+}\right)$it is apparent that fringe modulation is greater in the former spot.

As a consequence of the pupil selection, for each specklegram presented in Fig. 6 there is one pair of lateral diffraction spots where fringe visibility reaches its highest value. This is important because the zero order always contains the spectral information of both images and because high visibility is useful for accurate measurement of displacements in speckle photography. ${ }^{6,12}$

We note that the predicted fringe visibility for the zero order in the specklegrams in Fig. 6 is always less than unity, which is apparent when one compares the thick profiles for the zero order in Figs. 5 and 6 . Besides, fringe modulation for all those lateral spots whose visibilities reach their maximum is similar for the experiments illustrated in both figures.

\section{B. Multiple-Exposed Specklegrams}

Examples of interferometric fringes achieved by multiplexing three and four modulated speckle distributions are depicted in Figs. 7(a) and 7(b), respectively. Between images, diffuser uniform in-plane displacements were simulated. We obtained the images in Fig. 7 by following the same procedure as for Figs. 4-6. There, the respective normalized versions of the function $\ln \{1$ $+\left[I_{f}(U, V]^{1 / 2}\right\}$ are depicted.

For Fig. 7(a) we recorded three images by using the pupils $P^{1}, P^{2}$, and $P^{3}$ represented in Fig. 2 . Between the first and the second images, a horizontal $40-\mu \mathrm{m}$ diffuser displacement was simulated. A vertical $40-\mu \mathrm{m}$ diffuser displacement was simulated between the second and the third images also. Thus the third image was displaced

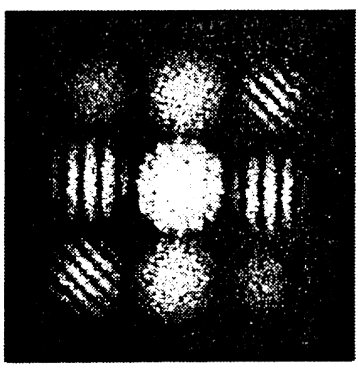

(a)

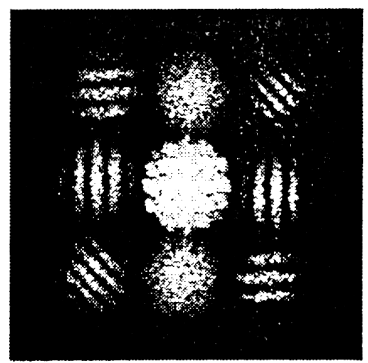

(b)
Fig. 7. (a) Triple-exposed and (b) quadruple-exposed specklegrams for different uniform in-plane displacements between images recorded through the pupils illustrated schematically in Fig. 2. 
both horizontally and vertically with respect to the first image. Then it holds that $\Delta X^{12}=\Delta X^{\text {n13 }}=\Delta Y^{13}=\Delta Y^{23}$ $\equiv \Delta$ and $\Delta Y^{12}=\Delta X^{23}=0$. Note that no fringes appear in the spot at $\left(d^{-}, d^{+}\right)$because only light diffracted by the pupil $P^{2}$ goes there $\left(q_{d^{-} d^{+}}^{1}=q_{d^{-} d^{+}}^{3}=0\right.$ and $\left.q_{d^{-} d^{+}}^{2}=1\right)$. Similarly, there are no fringes into the spot located at $\left(0, d^{+}\right)$. In this case the spectra of both the first and the second images go there $\left(q_{0 d^{+}}^{1}=q_{0 d^{+}}^{2}=1\right)$. Nevertheless, there are no common aperture pairs in the pupils $P^{1}$ and $P^{2}$ that are responsible for that spot formation $\left(q_{0 d^{+}}^{12}=0\right)$. For the spot at $\left(d^{+}, 0\right)$, only the two common apertures of the pupils $P^{1}$ and $P^{2}$ are responsible for the light diffracted into this spot $\left(q_{d^{+} 0}^{1}=q_{d^{+} 0}^{2}=q_{d^{+} 0}^{12}\right.$ $=1$ and $\left.q_{d^{+} 0}^{3}=q_{d^{+} 0}^{13}=q_{d^{+} 0}^{23}=0\right)$. It follows that the average intensity is $\left\langle I_{d^{+}}(U, V)\right\rangle=4 \cos ^{2}\left(\pi U \Delta / \lambda_{R} f\right) \kappa(U$ $-d^{+}, V$. Observe that fringe orientation corresponds to the horizontal displacement between the first and the second images and that the predicted visibility is optimum $\left(\mathcal{V}_{d^{+} 0}=1\right)$ for this spot. In a similar way we can interpret the fringes in the spot centered at $\left(d^{+}, d^{+}\right)$by considering that only the spectra of the first and the third images are overlapped into the spot. Also, the visibility reaches its highest value, but the period and the orientation of the fringes correspond to the relative displacement between the first and the third images. In this case the intensity is $\left\langle I_{d^{+} d^{+}}(U, V)\right\rangle=4 \cos ^{2}\left[\pi\left(U+V \Delta / \lambda_{R} f\right] \wedge(U\right.$ $\left.-d^{+}, V-d^{+}\right)$. Finally, in the central spot the spectra of the three images are overlapped $\left(q_{00}^{1}=q_{00}^{2}=3\right.$ and $q_{00}^{3}$ $=2$ ). Furthermore, a rather complex spatial modulation appears because horizontal, vertical, and diagonal fringes are also overlapped there $\left(q_{00}^{12}=q_{00}^{13}=2\right.$ and $q_{00}^{23}$ $=1$ ). From Eq. (33), the corresponding intensity is

$$
\begin{aligned}
\left\langle I_{00}(U, V)\right\rangle= & \left\{8+4 \cos \left(2 \pi U \Delta / \lambda_{R} f\right)\right. \\
& +2 \cos \left(2 \pi V \Delta / \lambda_{R} f\right) \\
& \left.+4 \cos \left[2 \pi(U+V) \Delta / \lambda_{R} f\right]\right\} \kappa(U, V) .
\end{aligned}
$$

In Fig. 7(b) a fourth image was added to the previous three images. We recorded the fourth image by using the pupil $P^{4}$ of Fig. 2. The third and fourth images were equally displaced with respect to the first; that is, no relative displacement between the last two images is of concern. Nevertheless, a new system of fringes appears in the spots centered at $\pm\left(d^{-}, d^{+}\right)$as a consequence of the overlapping of the spectral components of the second and the fourth images, exclusively. In fact, these (horizontal) fringes are related to the relative vertical displacement between the respective images. Also, the theoretical visibility value is 1 , and no change in the other lateral spots is introduced when the fourth image is added. Of course, the zero order contains spectral components that belong to the four images.

To obtain Fig. 8 we carried out real experiments. In this case, a conventional photographic procedure for recording the specklegrams and coherent-optical processing for analyzing them were implemented (instead of the digital procedures employed before). The diffuser was illuminated by a collimated $\mathrm{He}-\mathrm{Ne}$ laser beam $\left(\lambda_{W}\right.$ $=633 \mathrm{~nm}$ ) and imaged onto a high-resolution holographic film (Agfa-Gevaert 10E75). As before, the distances from the diffuser to the lens and from the lens to

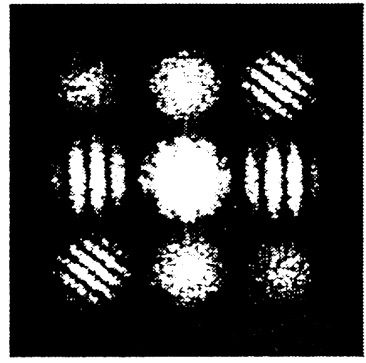

(a)

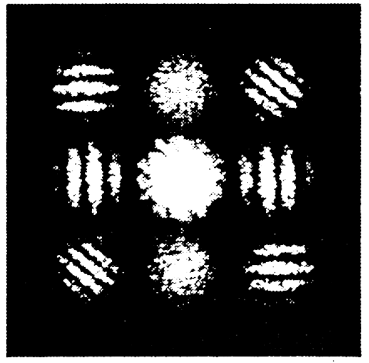

(b)
Fig. 8. (a) Triple-exposed and (b) quadruple-exposed real experiments for different uniform in-plane displacements between images.

the film were set to $Z_{0}=138 \mathrm{~mm}$ and $Z_{C}=382 \mathrm{~mm}$, respectively. For recording, the same sequential procedure and geometric parameters of the pupil masks were maintained. Between the first and the second and between the second and the third images a horizontal $40-\mu \mathrm{m}$ and a vertical $45-\mu \mathrm{m}$ in-plane diffuser displacement, respectively, was introduced. In Fig. 8(a) a three-exposure specklegram is shown; for Fig. 8(b) an additional exposure was made. There was no relative displacement between the third and the fourth images. To analyze the specklegrams we employed a coherent analogical Fouriertransform procedure. Observe that similar results are obtained in the real experiments and the simulated cases, as is apparent from a comparison of Figs. 7 and 8.

In summary, in this experiment the fringe-modulated lateral diffraction spots correspond to three independent double-exposed specklegrams with optimum visibility and for different relative displacements. Only four recording steps are needed, and relative displacements between nonconsecutive images are depicted. Note that because the last two images were displaced by the same amount in this experiment, redundancy in fringe information occurs in Fig. 8(b). However, this could be used to achieve more accurate fringe measurements. ${ }^{8}$

In the context of speckle photography we have restricted ourselves to demonstrating the application of uniform in-plane displacements. Nevertheless, a similar procedure could be carried out for analysis of a general inplane displacement distribution. To illustrate this, let us assume that four images are recorded with the same procedure as for Fig. 8(b), except that the diffuser is rotated between exposures. In this case no interference fringes will be observed at the transform plane when an expanded laser beam is employed to analyze the specklegram. However, two approaches (one pointwise and one whole field) are possible to measure local displacements. ${ }^{3}$

If we probe each small region of the specklegram point by point with a narrow laser beam, then straight equispaced Young fringes will be observed in the diffraction spectrum. ${ }^{12}$ Besides, as in Fig. 8(b), three different systems of fringes will appear in the lateral diffraction spots, depicting different displacements at the same region of the input plane. Note that this approach has no practical meaning in the case of a multiaperture arrangement through the same pupil for recording, as is pointed out in Ref. 8. 
On the other hand, isothetic fringes could be generated by optical filtering of specklegrams. In this case, an opaque mask with a small aperture is located onto a fringe-modulated diffraction spot, and light passing through the aperture is in turn Fourier transformed. ${ }^{8}$ In our approach light from different spots carries spectral information about different rotations, which implies that isothetics obtained from each spot will depict equal displacement components for a different in-plane rotation. Although it is beyond the scope of this paper, with a proper recording schedule with different multiaperture pupils it is possible to obtain in a single frame independent isothetic fringes of equal sensitivity and thus to measure different general in-plane displacement distributions.

\section{CONCLUSIONS}

We have proposed a multiplexing method for speckle photography that uses different multiple-aperture pupils for recording. It was shown that the introduction of proper spatial frequency carriers through the internal modulation of speckles allows selective concentration of the spectral components of individual or various images into isolated spots in the Fourier plane.

Both theoretical and experimental results obtained extend the speckle photography technique to depict simultaneously in a single frame several systems of interferometric fringes related to a series of uniform in-plane displacements between images. Also, a comparison of relative displacements between nonconsecutive recorded images is possible.

The cross-correlation functions of the amplitudes and intensities of speckled patterns generated by different pupils and by a diffuser uniform in-plane displacement between images were computed. On this basis, the correlation between images is determined by the spectral components of light admitted by the two pupils and displacements. Each pair of pupils can be associated with an independent contribution to fringe formation, which depends on the transmission area that the two pupils have in common.

The theoretical predictions thus obtained become a relevant tool for designing the recording routine and the set of pupils for the experiment to optimize fringe visibility for accurate measurements. Moreover, the highest visibility value is achieved, as when the same pupil is used for recording.

On the other hand, by proper selection of the different multiple-aperture pupils for recording, fringe-modulated speckle patterns with predetermined correlation properties and interferometric fringes with variable visibility can be generated.

The multiplexing technique with the use of different multiple-aperture pupils is not restricted to the transmission arrangement geometry; it could also be used for a properly illuminated rough reflecting surface.

Although in this paper we have analyzed only the use of different multiple-aperture pupils for uniform in-plane displacement measurements in speckle photography, applications can be implemented with this approach, for both real-time image processing ${ }^{11}$ and metrology. In particular, real-time speckle photography through recording by different multiple-aperture pupils for strain analysis is currently being investigated.

\section{ACKNOWLEDGMENTS}

This research was performed under the auspices of the Consejo Nacional de Investigaciones Cientificas y Técnicas, Comisión de Investigaciones Cientificas de la Provincia de Buenos Aires, Faculty of Engineering, National University of La Plata (Argentina). L. Angel acknowledges support from the Consejo Nacional de Investigaciones Científicas y Técnicas (Argentina) and Instituto Colombiano para el Desarrollo de la Ciencia y la Tecnologia, "Fransisco José de Caldas" (Colombia).

Address correspondence to $\mathrm{N}$. Bolognini at postmaster@ciop.edu.ar.

*Permanent address, Universidad Eafit, Medellin, Colombia.

${ }^{\dagger}$ Also with Facultad de Ingeniería, Universidad Nacional de La Plata, La Plata, Argentina.

${ }^{\ddagger}$ Also with Facultad de Ciencias Exactas, Universidad Nacional de La Plata, La Plata, Argentina.

\section{REFERENCES}

1. M. Françon, "Information processing using speckle patterns," in Laser Speckle and Related Phenomena, J. C. Dainty, ed. (Springer-Verlag, New York, 1975), pp. 171202.

2. I. Yamaguchi, "Speckle displacement and decorrelation in the diffraction and image fields for small object deformation," Opt. Acta 28, 1359-1376 (1981).

3. I. Yamaguchi, "Fringe formations in deformation and vibration measurements using laser light," in Progress in Optics, E. Wolf, ed. (North-Holland, Amsterdam, 1985), Vol. XXII, pp. 271-339.

4. D. W. Li, J. B. Chen, and F. P. Chiang, "Statistical analysis of one-beam subjective laser-speckle interferometry," J. Opt. Soc. Am. A 2, 657-666 (1985).

5. M. Sjödahl, "Calculation of speckle displacement, decorrelation, and object-point location in imaging systems," Appl. Opt. 34, 7998-8010 (1995).

6. G. H. Kaufmann, "Numerical processing of speckle photography data by Fourier transform," Appl. Opt. 20, 42774280 (1981).

7. R. Meynart, "Diffraction halo in speckle photography," Appl. Opt. 23, 2235-2236 (1984).

8. F. D. Chiang and R. P. Khetan, "Strain analysis by onebeam laser speckle interferometry. 2. Multiaperture method," Appl. Opt. 18, 2175-2186 (1979).

9. Y. Y. Hung, R. E. Rowlands, and I. M. Daniel, "Speckleshearing interferometric technique: a full-field strain gauge," Appl. Opt. 14, 618-622 (1975).

10. J. W. Goodman, "Statistical properties of laser speckle patterns," in Laser Speckle and Related Phenomena, J. C. Dainty, ed. (Springer-Verlag, New York, 1975), pp. 11-76.

11. L. Angel, M. Tebaldi, M. Trivi, and N. Bolognini, "Optical operations based on speckle modulation by using a photorefractive crystal," Opt. Commun. 168, 55-64 (1999).

12. K. Nakagawa, T. Takatsuji, and T. Minemoto, "Measurement of the displacement distribution by speckle photography using a BSO crystal," Opt. Commun. 76, 206-212 (1990).

13. K. Nakagawa and T. Minemoto, "Readout properties of the specklegram recorded in photorefractive $\mathrm{Bi}_{12} \mathrm{SiO}_{20}$ crystal," Appl. Opt. 30, 2386-2392 (1991).

14. S. Bosco and M. Trivi, "Correlation-like algorithm for superresolution analysis," Opt. Commun. 115, 444-448 (1995). 